\title{
MARKOV MODELING AND DISCRETE EVENT SIMULATION IN HEALTH CARE: A SYSTEMATIC COMPARISON
}

Lachlan Standfield, Tracy Comans, and Paul Scuffham

Centre for Applied Health Economics, School of Medicine \& Griffith Health Institute, Griffith University

Objectives: The aim of this study was to assess if the use of Markov modeling (MM) or discrete event simulation (DES) for cost-effectiveness analysis (CEA) may alter healthcare resource allocation decisions.

Methods: A systematic literature search and review of empirical and non-empirical studies comparing MM and DES techniques used in the CEA of healthcare technologies was conducted.

Results: Twenty-two pertinent publications were identified. Two publications compared MM and DES models empirically, one presented a conceptual DES and MM, two described a DES consensus guideline, and seventeen drew comparisons between MM and DES through the authors' experience. The primary advantages described for DES over MM were the ability to model queuing for limited resources, capture individual patient histories, accommodate complexity and uncertainty, represent time flexibly, model competing risks, and accommodate multiple events simultaneously. The disadvantages of DES over MM were the potential for model overspecification, increased data requirements, specialized expensive software, and increased model development, validation, and computational time.

Conclusions: Where individual patient history is an important driver of future events an individual patient simulation technique like DES may be preferred over MM. Where supply shortages, subsequent queuing, and diversion of patients through other pathways in the healthcare system are likely to be drivers of cost-effectiveness, DES modeling methods may provide decision makers with more accurate information on which to base resource allocation decisions. Where these are not major features of the cost-effectiveness question, MM remains an efficient, easily validated, parsimonious, and accurate method of determining the cost-effectiveness of new healthcare interventions.

Keywords systematic review, economic evaluation, Markov, discrete event simulation, DES

Healthcare systems have scarce resources and require choices to be made regarding the allocation of these resources to remain sustainable. Financial resource allocation can be made arbitrarily, through market mechanisms, or they may be guided through economic modeling and cost-effectiveness analyses (CEA). The latter approach aims to allocate finite financial resources such that health gain (generally at a population level) is maximized. In this way, scarce and constrained financial resources are used as efficiently as possible.

Economic models are valuable tools used to assist decision makers in the estimation of value for money of new health technologies. While empirical clinical research methods for determining the comparative effectiveness of new healthcare interventions are well developed, inevitably they do not capture all the details required to support a fully informed decision about the incremental cost-effectiveness of a new healthcare technology. While scientific experiments can conclude that there is not enough evidence available to support a hypothesis, decision makers cannot fail to act on the information available to them (1). Rejecting or postponing the reimbursement of a new technology is a decision to continue reimbursing current practice instead of the new technology. Therefore, economic modeling provides an important formal and transparent means by which decisions can be made in the face of inevitably incomplete data.

The results of economic evaluations of the same health technology in different jurisdictions can differ leading to seemingly incongruous reimbursement decisions across regions. Some of these variations simply reflect legitimate differences in the economic question being posed. For example, two economic analyses investigating the same healthcare technology may take different perspectives, one a societal and one a payer perspective, and may legitimately elicit different conclusions. In another instance, the cost of the comparator treatment may be different in one jurisdiction compared with another, again leading to different, but equally legitimate reimbursement decisions. In some situations there may be a paucity of good quality data available to populate an economic model which may lead to erroneous cost-effectiveness estimates. In some cases, differences between economic model predictions may simply be due to calculation errors. In others, there may be more subtle reasons why the results of economic models vary, including differences in the complexity of the models, different underlying modeling assumptions and the use of different modeling techniques.

For over a decade researchers have been discussing the comparative advantages and disadvantages of Markov cohort 
models (MM) and discrete event simulation (DES) when applied to questions of cost-effectiveness of new healthcare technologies (2). This article seeks to consolidate these comparisons by providing a systematic overview of the practical differences the use of these different modeling techniques may have on the results of CEA and the allocation of resources in financially constrained healthcare systems.

The MM was named after Andrey Andreyevich Markov, a Russian mathematician that introduced the Markov "chain" for the first time in 1906 (3). The MM is characterized by the Markov assumption, or memorylessness, whereby the probability of a given transition in the system is independent of the nature or timing of earlier transitions (4). An MM has a timehorizon which is separated into fixed time periods referred to as cycles. During each of these cycles, the cohort may transition between a finite number of health states according to appropriate probabilities (5). The accrual of costs and utility in the MM are determined by the number of cycles (i.e., the period of time) and the proportion of the cohort that reside in each health state over the time-horizon of the economic model. MM currently dominate the healthcare economic evaluation literature.

In contrast, DES first emerged in the 1950s and is now recognized as the most commonly used simulation technique in the field of operational research (6). DES has been used in a wide array of industries including: manufacturing, travel, finance, and health care. DES is characterized by individual entities (e.g., patients) that undergo a series of processes (events) that affect the entities attributes (such as outcomes) over time (7). Typically, a list of current or future events is constructed by randomly sampling time-to-event distributions for each event, placing them in the order in which the events are predicted to occur, and attaching them to each individual entity. In this way, each entity carries an event list describing the type and timing of current or future events which may occur to the entity over time. This event list may be updated after each event to reflect the impact the "history" of the entity has on current or future events. Unlike the MM, a DES does not have fixed equal cycle lengths; instead a DES is event driven and progresses according to the timing of these events. A DES also allows interaction between entities (e.g., patients) and resources (e.g., hospital beds) such that queues can form if the demand for resources exceeds supply.

The term MM is often poorly defined in the literature. This may lead to ambiguity in the interpretation of the relative benefits of MM when compared with other modeling techniques. To avoid such ambiguity, in this article, an MM is defined as a closed system Markovian, discrete-time state-transition model which calculates "expected value" results (i.e., a cohort analysis). This includes MM in which transition probabilities can change over the modeling period (e.g., by being indexed to the model cycle) and where temporary health states and tunnel states can be used. This definition excludes closed system discrete-time individual patient simulation (IPS) state-transition models, often referred to as a Monte-Carlo microsimulation, hereafter referred to as microsimulations. The term microsimulation used herein refers to microsimulation as generally defined in the health economics literature, which model individual patients one at a time, without interaction between patients. Like DES, microsimulation models allow individual patient history to be accrued during the simulation and for this history to inform a patient's future events and pathways through the modeled healthcare system.

\section{METHODS}

A systematic search of the literature was conducted capturing citations indexed up until the 5 December 2012. Medline records from 1950 onward and Embase records from 1947 onward were searched concurrently using EMBASE.com. The Cochrane Library, including the National Health Service Economic Evaluation Database (NHS EED), was also searched for pertinent literature. Only full publications reported in peer-reviewed journals which drew comparisons between MM and DES modeling techniques when used for CEA of healthcare technologies were included in the review.

The following literature search terms were used to identify pertinent literature: discrete event simulation (and synonyms such as DES, discrete event, event simulation), Markov, microsimulation, Monte-Carlo, cost and economic and synonyms thereof. The title and abstract of the article were reviewed by L.S. to determine the relevance of the article to the current review. Abstracts were excluded from the review if they were duplicates of other abstracts, were not full publications (e.g., letters, editorials, conference abstracts), were not focused on health economics, were not cost-effectiveness analyses, did not compare Markov and DES methods or were not published in English. If a publication could not be excluded on the basis of its abstract then the full publication was retrieved for detailed review. Two authors (L.S. \& T.C.) reviewed all included publications in full to identify the advantages and disadvantages reported for each modeling method. Any differences were reviewed by the third author (P.S.) and agreed by consensus.

\section{RESULTS}

A total of 584 citations were identified in the literature searches. Some thirty-five publications could not be excluded on the basis of their abstract alone and were retrieved in full. A total of twenty-two of these publications were pertinent to the final review. The reasons for excluding the remaining 562 citations are shown in Table 1.

Table 2 presents a brief description of each of the included publications and the methods by which conclusions were drawn on the relative advantages and disadvantages of MM and DES models. The majority of publications drew conclusions on the advantages and disadvantages of MM versus DES modeling through a general discussion of the author's experience and 
Table 1. Inclusion and Exclusion of Publications Comparing Discrete Event Simulation and Markov Modeling

\begin{tabular}{lc}
\hline Reason for exclusion & $\begin{array}{c}\text { No. of publications } \\
\text { excluded }\end{array}$ \\
\hline Duplicate articles & 25 \\
Abstract only & 48 \\
Non-English & 17 \\
Not a health economics publication & 84 \\
Not a cost-effectiveness analysis & 18 \\
Not a comparison of Markov modeling and discrete & 370 \\
$\quad$ event simulation & \\
Included publications & 22 \\
\hline
\end{tabular}

understanding of the modeling techniques (17 publications $(1 ; 2 ; 7-21)$, one study (two publications) described a consensus guideline on good modeling practices for DES $(22 ; 23)$, one publication presented a comparison of a conceptual MM and DES model (24), and the remaining two publications compared the results of an MM and DES model empirically $(25 ; 26)$. One of these studies compared the MM and DES model results with each other (25) and another compared the results of each model with each other and with an actual data-set (26).

The primary differences between MM and DES models as described in the identified publications are summarized in Table 3. Many of these differences are interrelated. The most commonly reported advantage of DES over MM was that DES modeling can track individual patient histories, such that each individual in the economic model can carry a large amount of information which can affect their future treatment options, risk of events and prognosis over time $(1 ; 7-12 ; 14 ; 17 ; 18 ; 21 ; 22 ; 24)$. The tracking of individual patient history also allows DES to capture individual risk factors that affect outcomes in a nonlinear manner $(7 ; 21)$. Furthermore, the tracking of individual patient history facilitates the modeling of interaction between covariates $(7 ; 11-13)$. In this way, a DES model may produce a realistic set of virtual patient histories (14). In comparison, MM has a more limited ability to capture patient history and nonlinearity. A solution to this in an MM can be to define more health states, use tunnel states or to further relax the Markovian assumption by using different transition matrices as time progresses $(7 ; 9 ; 11 ; 12)$. However, by definition, in an MM the "residents" of these health states are assumed to be homogenous patient groups. Therefore, an MM may need an enormous number of health states to represent complex systems and this may only be feasible for a limited number of items that need to be remembered $(7 ; 9 ; 12 ; 14)$.

Another commonly reported benefit of DES is its ability to allow the modeling of more complex systems than MM $(2 ; 10 ; 12 ; 14 ; 15 ; 24)$. This feature is partly related to the DES
Table 2. Description of Included Publications

Author (date) $\quad$ Focus of research

$\begin{array}{ll}\text { Review articles } & \\ \text { Barton (2004)(8) } & \text { Health care } \\ \text { Brennan (2006)(7) } & \text { Health technologies } \\ \text { Caro (2010)(9) } & \text { Health care } \\ \text { Cooper (2006)(10) } & \text { Coronary heart disease } \\ \text { Heeg (2008)(11) } & \text { Schizophrenia } \\ \text { Hollingworth (2007)(12) } & \text { Health care } \\ \text { Karnon and Brown (1998)(2) } & \text { Adjuvant treatment for breast cancer } \\ \text { Weinstein (2006)(1) } & \text { Health care } \\ \text { Systematic reviews } & \\ \text { Hughes (2007)(13) } & \text { Compliance \& persistence modeling } \\ \text { Kamal (2006)(14) } & \text { TNF alpha for rheumatoid arthritis } \\ \text { Kim and Goldie (2008)(15) } & \text { Vaccination programs } \\ \text { Articles describing a DES model alone } & \\ \text { Caro (2005)(16) } & \text { Bipolar mood disease } \\ \text { Heeg (2005)(17) } & \text { Schizophrenia } \\ \text { Karnon and Brown (2002)(18) } & \text { Early breast cancer } \\ \text { Lindgren (2009)(19) } & \text { Rheumatoid arthritis } \\ \text { Tran-Duy (2011)(21) } & \text { Ankylosing spondylitis } \\ \text { Xenakis 2011 (20) } & \text { Smoking cessation } \\ \text { Consensus guideline } & \\ \text { Karnon (2012) (22, 23) } & \text { Health care } \\ \text { Articles describing a comparison of a MM } & \\ \text { and a DES } & \\ \text { Karnon (2003)(25) } & \text { Empirical comparison of DES and MM in } \\ & \text { early breast cancer } \\ \text { Le Lay (2006)(24) } & \text { Conceptual comparison of MM and DES } \\ \text { Simpson (2009) (26) } & \text { in depression } \\ & \text { Empirical comparison of DES and MM in } \\ \text { human immunodeficiency virus }\end{array}$

DES, discrete event simulation; MM, Markov model; TNF, Tumour necrosis factor.

modeling methods ability to track individual patient attributes (10). However, the flexibility of DES is also supported its ability to model interactions between patients, or between patients and the environment $(7 ; 10 ; 12 ; 15 ; 22)$. This feature is often used when modeling queuing systems, where supply and demand unfolds probabilistically over time (1). This allows DES to capture situations when constraints on resources mean that the choice of treatment for one patient affects what can be given to another $(8 ; 22 ; 23)$. These changes may affect the system performance in a nonlinear manner which is accommodated by DES methods (7). It is not possible to capture such patient interactions and queuing using an MM.

Several authors suggest DES is well suited to modeling situations where patients are subject to multiple competing risks 
Standfield et al.

Table 3. Description of Advantages and Disadvantages of Markov versus DES Models

\begin{tabular}{|c|c|c|}
\hline Attribute & Comparison of Markov and DES modeling & Sources \\
\hline \multicolumn{3}{|l|}{ Content issues } \\
\hline Memory, individual patient history & $\begin{array}{l}\text { MM have a limited ability to capture patient history (ie. due to the Markovian } \\
\text { assumption). As an IPS method DES can account for the impact of individual patient } \\
\text { history on future events. }\end{array}$ & $(1,7-12,14,17,18,21,22,24$ \\
\hline Accommodate complexity & Higher levels of complexity can be accommodated with DES. & $(2,10,12,14,15,24)$ \\
\hline $\begin{array}{l}\text { Interaction between individuals, } \\
\text { individuals and resources and } \\
\text { queuing }\end{array}$ & $\begin{array}{l}\text { This is not possible in MM. DES can simulate interactions amongst individuals or between } \\
\text { individuals and the environment. This is useful in simulating complex systems involving } \\
\text { queves and delays due to competition for limited resources. }\end{array}$ & $(7,10,12,15,22)$ \\
\hline Modeling competing risks & $\begin{array}{l}\text { DES is well suited to modeling situations where patients are subject to multiple or } \\
\text { competing risks. A DES manages the competing and the sequencing of events by } \\
\text { generating a future events list, then, for example, selecting the next closest } \\
\text { time-to-event to ascertain which event occurs next in the process. In an MM a transition } \\
\text { probability is derived for each mutually exclusive competing health state and these } \\
\text { competing health states must be exhaustive. }\end{array}$ & $(12,16,22,23)$ \\
\hline $\begin{array}{l}\text { Ability to manage multiple events at } \\
\text { the same time }\end{array}$ & $\begin{array}{l}\text { In an MM the cohort is in one of a variety of mutually exclusive health states at any one } \\
\text { time. In a DES events are instantaneous and, therefore, can occur simultaneously. }\end{array}$ & $(9,11,12,17,24)$ \\
\hline Time representation & $\begin{array}{l}\text { MM have a fixed cycle length. In DES the time between events can be of any duration } \\
\text { and may vary throughout the simulation. }\end{array}$ & $(12,16,22,23,26)$ \\
\hline Ability to capture uncertainty & $\begin{array}{l}\text { DES models can capture a greater level of detail than MM allowing the model to capture } \\
\text { more detail regarding uncertainty in the system being modeled. }\end{array}$ & $(2,11,24)$ \\
\hline Face validity and realism of model & $\begin{array}{l}\text { DES may predict the course of a disease more naturally than an } M M \text { and this may } \\
\text { increase the face validity of the economic model. }\end{array}$ & $(17,26)$ \\
\hline \multicolumn{3}{|c|}{ 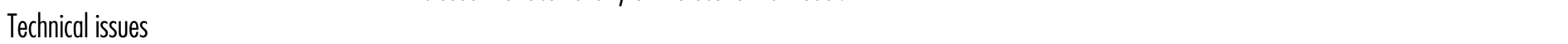 } \\
\hline Development time & DES models are generally more complex and take more time to develop than MM. & $(2,10,14,15,24,25)$ \\
\hline Validation time & DES models are generally more complex and time consuming to validate than MM. & $(2,10,14,15,24,25)$ \\
\hline Computational time & Increased with DES compared to MM. & $(7,11,15)$ \\
\hline Model over-specification & $\begin{array}{l}\text { DES may induce overspecification where models may become more complex than } \\
\text { necessary. }\end{array}$ & $(2,24)$ \\
\hline Data requirements & Generally increased with DES compared to MM. & $(2,9,11,12,15,20,24,26)$ \\
\hline Specialist software and analyst input & DES requires specialised, often expensive software. & $(2,8,12,16,22-24)$ \\
\hline
\end{tabular}

DES, discrete event simulation; IPS, individual patient simulation; MM, Markov model; SR, systematic review.

$(12 ; 16 ; 22 ; 23)$. A DES manages the competing and the sequencing of events by generating a future events list, then, for example, selecting the next closest time-to-event to ascertain which event occurs next in the process. Then this process is repeated and any impact the updated patient history may have on future events is captured. Alternatively, as recommended in the consensus guidelines by Karnon et al. 2012, the analyst may sample the time to one of a range of competing events or risks and then determine through another algorithm the event of interest that occurs (22;23). In an MM, a transition probability is derived for each mutually exclusive competing health state and these competing health states must be exhaustive. The incorporation of complexity is further enhanced by the DES model's ability to accommodate multiple events simultaneously $(9 ; 11 ; 12 ; 17 ; 24)$. In $\mathrm{MM}$, only one transition can be modeled per cycle. However, the impact of this limitation on accuracy may be reduced by decreasing the cycle length of the MM $(7 ; 11)$. Furthermore, authors note that the handling of time is explicit in DES $(12 ; 16 ; 22 ; 23)$. In an MM, a fixed discrete cycle length is used, which is not required in DES modeling where time can be varied throughout the duration of the simulation $(12 ; 16 ; 22 ; 23 ; 26)$.

As DES models are able to accommodate more complexity a greater level of detail and variables can be captured in these models than in MM. This greater flexibility allows these models to capture more detail regarding uncertainty in the system being modeled $(2 ; 11 ; 24)$. The additional variables can then be subject to probabilistic analysis. The corresponding sensitivity analyses may then give a better understanding of the uncertainty that exists in the base case values than in simpler models where these assumptions may remain implicit, not 
Table 4. Results of the Empirical Comparisons of Markov Modeling and Discrete Event Simulation

\begin{tabular}{|c|c|c|c|c|c|c|}
\hline Publication & Cost & $\mathrm{IC}$ & QALYS & IE & $\begin{array}{c}\text { CER } \\
\text { (Cost/QALY) }\end{array}$ & $\begin{array}{c}\text { ICER } \\
\text { (Cost/QALY saved) }\end{array}$ \\
\hline \multicolumn{7}{|c|}{$\begin{array}{l}\text { Simpson et al., } 2009 \text { (26) } \\
\text { MM }\end{array}$} \\
\hline Lop+Rit & USD 310,194 & & 10.55 & & USD 29,402 & \\
\hline $\begin{array}{l}\text { Ata+Rit } \\
\text { DES }\end{array}$ & USD 318,882 & -USD 8688a & 10.11 & $0.44^{a}$ & USD 31,541 & Lop+Rit dominant ${ }^{a}$ \\
\hline Lop+Rit & USD 340,022 & & 12.40 & & USD 27,421 & \\
\hline Ata+Rit & USD 352,843 & -USD $12,821^{a}$ & 12.11 & $0.29^{a}$ & USD 29,136 & Lop+Rit dominant ${ }^{a}$ \\
\hline \multicolumn{7}{|c|}{$\begin{array}{l}\text { Karnon } 2003(25) \\
\text { MM }\end{array}$} \\
\hline $\operatorname{Tam}+\mathrm{Cx}$ & $£ 8740$ & & 12.00 & & $£ 728^{a}$ & \\
\hline $\begin{array}{l}\text { Tam alone } \\
\text { DES }\end{array}$ & $£ 6721$ & $£ 2019^{a}$ & 11.40 & $0.60^{a}$ & $£ 589^{\circ}$ & $£ 3365$ \\
\hline $\operatorname{Tam}+\mathrm{Cx}$ & $£ 9146$ & & 12.14 & & $\mathfrak{£} 753^{a}$ & \\
\hline Tam alone & $£ 7115$ & $£ 2031^{a}$ & 11.56 & $0.58^{a}$ & $£ 615^{a}$ & $£ 3483$ \\
\hline
\end{tabular}

Note. NB. Rounding applies.

"Calculated post hoc.

Ata, atazanavir; CER, cost-effectiveness ratio; Cx, chemotherapy; DES, discrete event simulation; ICER, incremental cost-effectiveness ratio; IC, incremental cost; IE, incremental effect; Lop, lopinavir; MM, Markov model; QALY, quality-adjusted life-year; Rit, ritonavir; Tam, tamoxifen; USD, United States Dollar

quantified and consequently incontestable (11). DES also facilitates structural sensitivity analysis by allowing the incorporation of alternative model structures in the one simulation $(7 ; 16)$.

Some authors have also suggested that DES predicts the course of a disease more naturally than an MM and may, therefore, give superior "face validity" with decision makers $(17 ; 26)$. Many of the authors found that DES models were more complex and took more time to develop and to validate than $\operatorname{MM}(2 ; 10 ; 14 ; 15 ; 24 ; 25)$. Karnon and colleagues concluded that the slight benefit of DES, in terms of increased flexibility, were outweighed by the far greater time required to develop and evaluate the DES model (25). Furthermore, computational time was increased for DES over an MM $(11 ; 15)$. DES also has the additional disadvantage of potential computational slowness associated with inserting and removing events from the future event lists (7).

Some authors note that the flexibility of DES may lead to model over-specification, whereby possible patient models may become more complex than necessary $(2 ; 24)$. Furthermore, data requirements are generally increased with DES compared with MM $(2 ; 9 ; 11 ; 12 ; 15 ; 20 ; 24 ; 26)$. Also, unlike MM, authors note that DES models require specialized and often expensive software, with archaic programming conventions $(8 ; 26)$. Other researchers also cite the need for specialized analytical knowledge as a disadvantage of DES models $(2 ; 8 ; 24)$. The need for this specialist analytic knowledge may reduce accessibility to DES models $(12 ; 24)$. However, other authors note that there are now several high-level software packages available that may overcome this problem (e.g., Arena $\AA$, Simul8 $\cap$ ) $(16 ; 22 ; 23)$.

\section{Empirical Studies}

As previously stated, MM and DES were compared in two empirical studies $(25 ; 26)$. The results of these comparisons are presented in Table 4. In the study by Simpson, the results of the MM and DES were similar when the time-frame was short (1-year) but the DES model had a slightly better long-term (5year) predictive validity than the MM for HIV. However, the performance of the MM was good, with the clinical outcomes generated by the model falling within a 3 percent margin of error of the actual clinical data. The cost and effect estimates generated by the DES for both treatments (lopinavir/ritonavir versus atazanavir/ritonavir) were higher than those predicted by the MM. For the lopinavir/ritonavir regimen, the estimated cost-effectiveness ratio (CER) was around $\$ 2,000$ per qualityadjusted life-year (QALY) higher for the MM compared with the result obtained through DES. For the atazanavir/ritonavir regimen similar differences in the CER from the two competing models were found. Both models predicted cost-savings and QALY benefits for the lopinavir/ritonavir regimen over the 
atazanavir/ritonavir regimen (i.e., lopinavir/ritonavir dominates the atazanavir/ritonavir regimen). The authors found that DES was able to accommodate direct input of a range of patient inputs (CD4+ and plasma viral load levels) and provide many more details about what may be expected to happen in a population than the MM. Due to the limitations of MM methods the authors state that researchers tend to use categorical groupings to represent complex interacting continuous measures which may cause short-term aggregation bias leading to long-term prediction errors. In contrast, DES allows the inclusion of individual variables without the need to create compound, aggregate health states, which improves model precision (26).

These factors led the authors to conclude that DES represented the course of disease more naturally with fewer restrictions which may give the model superior face validity. Furthermore, they concluded that DES is better than MM in isolating long-term implications of small but important differences in crucial input data. However, the authors note that DES requires more data, data analysis, and programming time than an MM, although the authors acknowledge that the extra time required for the DES model implementation may be due to their lesser experience working with DES software. The authors also discuss the importance of transparency of the modeling methods to decision makers; however, on the basis of this study they could not conclude which of the two modeling methods was more transparent.

The second empirical study by Karnon and colleagues compared an MM and DES for the treatment of early breast cancer (25). Many of the underlying assumptions used in the two models were similar (e.g., disease free interval calculations, toxicity, etc). A total of one hour computational time was required to run the MM while the DES model took 3 days to run. Like the findings in the publication by Simpson, the DES model generated higher cost and effect estimates for both treatments than the MM, but both modeling methods predicted very similar incremental cost-effectiveness ratio results. Nonetheless, the authors found that the closeness of these results disguised some potentially important differences in the models that appear to have balanced each other out. The comparison of the alternative modeling techniques identified two areas in which DES facilitated a more flexible representation of the available data (i.e., state entry-dependent probabilities and set survival times). However, the authors found that the process of verification and validation for DES was longer, taking weeks, compared to days for the MM. The authors acknowledged that when estimating the time required to build, verify, and validate a model, factors such as experience with each method should be accounted for.

The authors concluded that the increased flexibility of DES may only provide significant improvements in the accuracy of a modeling evaluation when the areas of increased flexibility apply to a large proportion of the model. In the example presented, the authors found that the closeness of the results of the MM and DES model suggested that it was unlikely that the use of one model's results over the other would lead to an alternative resource allocation decision, and posited that the slight benefits of DES, in terms of increased flexibility, were outweighed by the far greater time required to develop and evaluate the DES model (25). Therefore, the authors concluded that, in this instance, the MM was the optimal technique for evaluation of alternative adjuvant therapies for early breast cancer. However, the authors also acknowledged that there may be circumstances in which a DES model would provide a more accurate representation of the available data.

\section{DISCUSSION}

The evidence comparing MM and DES suggests that DES is a useful addition to the modeler's armamentarium for use in CEA of healthcare interventions in certain situations. One of the key advantages of DES over MM is that, like microsimulation, it is an IPS technique. This allows individual patient history to be accrued during the simulation and for this history to inform a patient's future events and pathways through the modeled healthcare system. IPS techniques accommodate correlations, inter-related covariates and nonlinear effects of patient history on future events in a more flexible manner than MM. However, this increased flexibility may lead to model over-specification, where economic models may become more complex than is necessary to elicit an accurate result. Furthermore, while not a necessity, data requirements are generally increased with DES compared with MM. Also DES is generally more complex and may require more specialist skills and take more time to develop and validate compared with MM. These additional requirements may increase the time required for governments to independently verify the results of such analyses and may reduce the apparent transparency of such analyses to decision makers. In some circumstances, the potential for increased model complexity and data requirements may act as a barrier to the adoption of DES over MM.

In practice, the MM has evolved well beyond the strict confines of the Markovian assumption. Using additional health states, tunnel states, and temporary health states allows the analyst to manipulate the Markovian assumption to capture the history of the cohort. This is further enhanced through the use of transition probabilities that are indexed to the number of cycles the cohort has spent in the model or in a particular tunnel state. However, as the interplay between patient history and future events increases in complexity the number of the additional health states required to capture this complexity increases exponentially. In some cases, this will lead to a situation where an MM becomes unwieldy and ultimately unsuitable for the accurate modeling of the cost-effectiveness question at hand. In these cases, the use of a microsimulation or a DES model would appear appropriate.

DES uses a future events list for each modeled entity which highlights an important conceptual difference between a DES 
and MM. A DES manages the competing and the sequencing of events by generating a future events list, then, for example, selecting the next closest time-to-event to ascertain which event occurs next in the process. Then this process is repeated and any impact the updated patient history may have on future events is captured. The rationale for this approach is that the current event may alter the patient's probability of a subsequent event. This approach provides a relatively simple way to manage a multitude of competing events and schedule them. In contrast, in an $\mathrm{MM}$ and a microsimulation a transition probability is derived for each mutually exclusive competing health state and these competing health states must be exhaustive.

Another area which clearly distinguishes the MM and microsimulation modeling approaches from DES is that DES allows interaction between patients or between patients and the environment whereas MM and microsimulation does not $(7 ; 10 ; 12 ; 15)$. This allows DES to capture situations when constraints on resources mean that the choice of treatment for one patient affects what can be given to another (8). Furthermore, these changes may affect the system performance in a nonlinear manner which is accommodated by DES methods (7). However, the analyst should consider whether any incremental change in demand introduced by the new intervention being modelled is likely to lead to the alleviation or exacerbation of supply shortages and changes in subsequent queuing, or diversion of patients through other, often less efficient, pathways in the health care system. Where this is not the case, or this is unlikely to be a driver of cost-effectiveness, incorporating queuing into the economic model is likely to be an unnecessary over-specification. In other situations, such as the modeling of demand for nursing home care for patients with Alzheimer's disease or other dementias in increasingly ageing populations, the ability of an economic model to capture the impact of competition for resources may be crucial. The choice of modeling method in this situation has the potential to result in very different resource allocation decisions that may alter the overall efficiency of the healthcare system markedly.

A MM is a cohort level analysis that only needs to be run once to generate expected values of cost and effect. This process is generally very rapid. In comparison, an IPS such as DES requires that multiple iterations of the model be calculated to generate accurate and precise estimates of the expected values of cost and effect. Clearly this leads to increased computational time. However, in theory, a DES model may be more computationally efficient than a comparable microsimulation. In a microsimulation, each patient must traverse all of the cycles of the model, if the model is constructed using a lifetime time horizon and the cycle length is short this may lead to a high computational burden. In contrast, in a DES model, only pertinent events are modeled and as time is explicit and stochastic a patient may traverse the model in a small number of calculations, thereby decreasing computational burden. In some situations, this may lead to the use of a DES over a microsim- ulation. However, the computational efficiency of DES, when compared with microsimulation, may be somewhat tempered as a DES must maintain a future event list where events must be inserted and removed as required (7). Furthermore, in CEA, the economic model must ascertain when costs and effects are being accrued so that they may be discounted appropriately. This may require that the DES model is supplemented with additional calculations to assess the timing of cost and effect accrual and allow appropriate discounting calculations to be applied. Furthermore, the efficiency of the programming used should also be considered as this can have an impact on computational times. Regardless, as many authors discuss, the availability of relatively inexpensive computational power and efficient computer programs to run such analyses can somewhat alleviate these computational burden considerations.

This review has demonstrated the advantages and disadvantages of MM and DES. However, this review is not without limitations. While a substantial number of articles were identified which provide important insights into the advantages and disadvantages of DES compared with MM, the majority of publications identified compared MM and DES subjectively through the authors' experience and understanding of the modeling techniques. Only three studies were identified that compared MM and DES models directly (24-26) and one of these studies was a comparison of conceptual models only (24). The remaining two studies compared DES and MM empirically $(25 ; 26)$, however, only one of these studies attempted to calibrate these results back to an actual dataset (26). Both studies found that DES took longer to develop and validate than the MM. However, the authors acknowledged that when estimating the time required to build, verify, and validate a model factors such as experience with each method should be accounted for. In the two empirical analyses, both modeling techniques provided similar guidance on the incremental cost-effectiveness of the products being investigated. However, both studies found that the DES technique was able to accommodate higher levels of complexity than an $\mathrm{MM}$ and, in some situations, this may result in DES being more accurate than an $\mathrm{MM}(25 ; 26)$. Also, it is important to note that, while these studies explored the ability of DES and MM to accommodate complexity, neither of the studies focused on the potential differences in cost-effectiveness results that may be driven by queuing and competition for limited resources. This is an area that needs to be researched in more detail.

In summary, DES modeling, like other IPS methods, would be preferred over MM where individual patient history is an important and complex driver of future events. Furthermore, DES would appear to be superior to MM when modeling situations where supply shortages and subsequent queuing, or diversion of patients through other, often less efficient, pathways in the healthcare system are likely to be a driver of cost-effectiveness. Where this is not the case, incorporating queuing into the economic model is likely to be an unnecessary over-specification which is unlikely to be informative to decision makers. 
Additionally, DES may have some advantages over MM when modeling patients at multiple competing risks. However, where these are not major features of the cost-effectiveness question, it would appear that the MM remains an efficient, transparent, easily validated, parsimonious and accurate method of determining the cost-effectiveness of new healthcare interventions. Therefore, MM remains an important tool by which to guide decision makers on the efficient allocation of scarce resources in the healthcare setting.

\section{CONTACT INFORMATION}

Lachlan Standfield, BBiotech (Hons I) (lachlan.standfield@, griffithuni.edu.au), Tracy Comans, PhD, Senior Research Fellow, Paul Scuffham, PhD, Professor and Director of Population and Social Health Research, Centre for Applied Health Economics, School of Medicine \& Griffith Health Institute, Logan Campus, Griffith University, Meadowbrook, Queensland 4131, Australia

\section{CONFLICTS OF INTEREST}

The authors report no conflicts of interest.

\section{REFERENCES}

1. Weinstein MC. Recent developments in decision-analytic modelling for economic evaluation. Pharmacoeconomics. 2006;24:1043-1053.

2. Karnon J, Brown J. Selecting a decision model for economic evaluation: a case study and review. Health Care Manag Sci. 1998;1:133-140.

3. Kouemou G. History and theoretical basics of hidden Markov Models. In: Dymarski P, ed. Hidden Markov models, theory and applications. University Campus STeP Ri Slavka Krautzeka 83/A 51000 Rijeka, Croatia: InTech; 2011.

4. Drummond M, Sculpher M, Torrance G, O'Brien B, Stoddart G. Economic evaluation using decision analytic modelling. Methods for the economic evaluation of health care programs, 3rd ed. Oxford: Oxford University Press; 2005.

5. Sonnenberg FA, Beck JR. Markov models in medical decision making: A practical guide. Med Decis Making. 1993;13:322-338.

6. Hollocks B. Forty years of discrete-event simulation: A personal reflection. J Oper Res Soc. 2006;57:1383-1399.

7. Brennan A, Chick SE, Davies R. A taxonomy of model structures for economic evaluation of health technologies. Health Econ. 2006;15:12951310 .

8. Barton P, Bryan S, Robinson S. Modelling in the economic evaluation of health care: Selecting the appropriate approach. J Health Serv Res Policy. 2004;9:110-118.

9. Caro JJ, Moller J, Getsios D. Discrete event simulation: The preferred technique for health economic evaluations? Value Health. 2010;13:10561060 .
10. Cooper K, Brailsford SC, Davies R, Raftery J. A review of health care models for coronary heart disease interventions. Health Care Manag Sci. 2006;9:311-324.

11. Heeg BMS, Damen J, Buskens E, et al. Modelling approaches: The case of schizophrenia. Pharmacoeconomics. 2008;26:633-648.

12. Hollingworth W, Spackman DE. Emerging methods in economic modeling of imaging costs and outcomes. A short report on discrete event simulation. Acad Radiol. 2007;14:406-410.

13. Hughes D, Cowell W, Koncz T, Cramer J. Methods for integrating medication compliance and persistence in pharmacoeconomic evaluations. Value Health. 2007;10:498-509.

14. Kamal KM, Miller LA, Kavookjian J, Madhavan S. Alternative decision analysis modeling in the economic evaluation of tumor necrosis factor inhibitors for rheumatoid arthritis. Semin Arthritis Rheum. 2006;36:5060.

15. Kim SY, Goldie SJ. Cost-effectiveness analyses of vaccination programmes: A focused review of modelling approaches. Pharmacoeconomics. 2008;26:191-215.

16. Caro JJ. Pharmacoeconomic analyses using discrete event simulation. Pharmacoeconomics. 2005;23:323-332.

17. Heeg BM, Buskens E, Knapp M, et al. Modelling the treated course of schizophrenia: Development of a discrete event simulation model. Pharmacoeconomics. 2005;23(Suppl 1):17-33.

18. Karnon J. Tamoxifen plus chemotherapy versus tamoxifen alone as adjuvant therapies for node-positive postmenopausal women with early breast cancer: A stochastic economic evaluation. Pharmacoeconomics. 2002;20:119-137.

19. Lindgren P, Geborek P, Kobelt G. Modeling the cost-effectiveness of treatment of rheumatoid arthritis with rituximab using registry data from Southern Sweden. Int J Technol Assess Health Care. 2009;25:181-189.

20. Xenakis JG, Kinter ET, Ishak KJ, et al. A discrete-event simulation of smoking-cessation strategies based on varenicline pivotal trial data. Pharmacoeconomics. 2011;29:497-510.

21. Tran-Duy A, Boonen A, Van De Laar MAFJ, Franke AC, Severens JL. A discrete event modelling framework for simulation of long-term outcomes of sequential treatment strategies for ankylosing spondylitis. Ann Rheum Dis. 2011;70:2111-2118.

22. Karnon J, Stahl J, Brennan A, et al. Modeling using discrete event simulation: A report of the ISPOR-SMDM Modeling Good Research Practices Task Force-4. Med Decis Making. 2012;32:701-711.

23. Caro JJ, Briggs AH, Siebert U, Kuntz KM, Force I-SMGRPT. Modeling good research practices-overview: A report of the ISPOR-SMDM Modeling Good Research Practices Task Force-1. Med Decis Making. 2012;32:667-677.

24. Le Lay A, Despiegel N, Francois C, Duru G. Can discrete event simulation be of use in modelling major depression? Cost EffResour Alloc. 2006;4:111.

25. Karnon J. Alternative decision modelling techniques for the evaluation of health care technologies: Markov processes versus discrete event simulation. Health Econ. 2003;12:837-848.

26. Simpson KN, Strassburger A, Jones WJ, Dietz B, Rajagopalan R. Comparison of Markov model and discrete-event simulation techniques for HIV. Pharmacoeconomics. 2009;27:159-165. 